\title{
Idiopathic Portal Hypertension in a Patient with Mixed Connective Tissue Disease and Protein $\mathrm{C}$ Deficiency
}

\author{
Yoshiro Horai, Tomoya Miyamura, Akie Hirata, Masataka Nakamura, Soichiro Takahama, \\ Hitoshi Ando, Rumi Minami, Masahiro Yamamoto and Eiichi Suematsu
}

\begin{abstract}
We report a 29-year-old woman with a 2.5 year history of mixed connective tissue disease (MCTD) who developed idiopathic portal hypertension (IPH) and thrombocytopenia as a result of hypersplenism. She had recurrent esophagogastric variceal rupture. Hematological examination also revealed low levels of protein $\mathrm{C}$ activity. The liver biopsy specimen showed non-specific mild inflammation and no thrombi. However, portal vein thrombosis developed after splenectomy. This was a rare case of severe complications of IPH accompanying MCTD and protein C deficiency.
\end{abstract}

Key words: idiopathic portal hypertension, mixed connective tissue disease, protein C deficiency

(Inter Med 49: 2013-2016, 2010)

(DOI: 10.2169/internalmedicine.49.3834)

\section{Introduction}

Mixed connective tissue disease (MCTD) is an autoimmune disease characterized by symptoms of three connective tissue diseases; systemic lupus erythematosus, systemic sclerosis and polymyositis. Idiopathic portal hypertension (IPH) is a disorder characterized by non-cirrhotic portal hypertension, pancytopenia and splenomegaly. Although the pathogenesis of IPH is still unknown, IPH is believed to have an association with immunological abnormalities $(1,2)$. However, IPH associated with MCTD is rare (3-8).

Protein $\mathrm{C}$ is a physiological anticoagulant, and activated protein $\mathrm{C}$ is thought to be an important factor in reducing endothelial dysfunction. Protein C deficiency (PCD) is recognized as a cause of various vascular diseases, including portal vein thrombosis. Persistently elevated inflammatory cytokine levels in MCTD are thought to be associated with a high risk of endothelial damage (9). However, there are no reports of an association between MCTD and PCD, and any interaction between MCTD and PCD on endothelial dysfunction has not been reported. We herein describe a 29year-old woman with IPH accompanied by MCTD and PCD.

Case Report

A 29-year-old woman was diagnosed with MCTD in April 2007, based on the presence of Raynaud's phenomenon, detection of anti-U1-ribonucleoprotein antibody, polyarthralgia, facial erythema, leukocytopenia, thrombocytopenia and sclerodactyly. After the diagnosis of MCTD was established, splenomegaly was detected on ultrasound examination. Steroid therapy with a medium dose was initiated. After the start of steroid therapy, mild fever disappeared and slightly elevated CRP levels became normal, whereas highly elevated erythrocyte sedimentation rate (ESR) (about 60 $\mathrm{mm} /$ hour) persisted, polyarthralgia was refractory to prednisolone and finger X-rays revealed progressive bone destruction. We began infliximab (IFX) and methotrexate $(8 \mathrm{mg} /$ week) in December 2008 and her arthralgia gradually improved. A $200 \mathrm{mg}$ dose of IFX was given intravenously after 2, 6 and 14 weeks and every 8 weeks thereafter. Administration of IFX continued without the patient suffering a relapse of polyarthralgia or side effects of IFX. ESR levels decreased, however, slightly elevated levels (about $40 \mathrm{~mm} /$ hour) persisted. She received IFX administration for the seventh time in August 2009, and was admitted to our department for the eighth IFX administration in late October 2009.

Department of Internal Medicine and Rheumatology, Clinical Research Center, National Hospital Organization Kyushu Medical Center, Fukuoka Received for publication April 20, 2010; Accepted for publication June 30, 2010

Correspondence to Dr. Yoshiro Horai, yoshirohorai0518@qmed.hosp.go.jp 


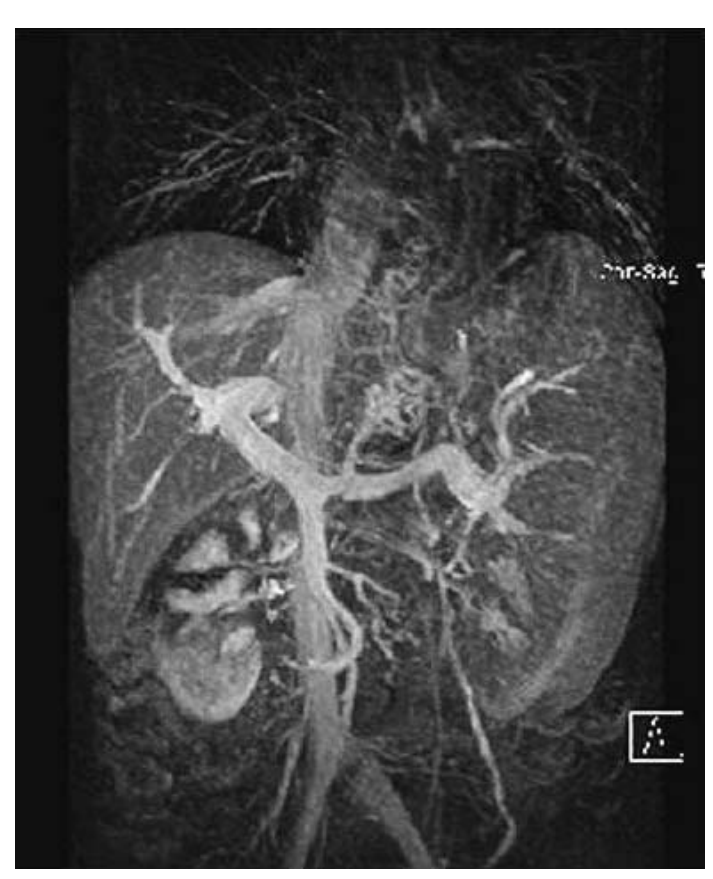

Figure 1. Magnetic resonance imaging shows dilation of the extrahepatic and intrahepatic portal veins and collateral veins, and severe splenomegaly.

However, 4 days before the admission, tarry stools had appeared. Her laboratory studies on the admission showed pancytopenia (white blood cell count, $3,400 / \mu \mathrm{L}$ (neutrophils $82.0 \%$, lymphocytes $14.0 \%$, monocytes $3.0 \%$, basophils $1.0 \%$ ); red blood cell count, $2.27 \times 10^{6} / \mu \mathrm{L}$; hemoglobin, 6.5 $\mathrm{g} / \mathrm{dL}$; hematocrit, 20.2\%), thrombocytopenia (platelet count, $7.9 \times 10^{4} / \mu \mathrm{L}$ ) and low levels and little activity of the protein $\mathrm{C}$ antigen (protein $\mathrm{C}$ antigen level, 37\%; protein $\mathrm{C}$ activity, $39 \%$ ). The levels of protein $\mathrm{S}$ activity and antithrombin III were normal (protein S activity, 75\%; antithrombin III, $96 \%)$. Slightly prolonged prothrombin time or activated partial thromboplastin time were present (prothrombin time, 16.8 seconds; international normalized ratio, 1.45; activated partial thromboplastin time, 36.2 seconds). Liver function tests revealed only mild enzyme elevation (aspartate aminotransferase, $50 \mathrm{IU} / \mathrm{L}$; alanine aminotransferase, $47 \mathrm{IU} /$ $\mathrm{L}$; alkaline phosphatase, $183 \mathrm{IU} / \mathrm{L} ; \gamma$-glutamyl transpeptidase, 36 IU/L). Serum hepatitis B surface antigen and antihepatitis $\mathrm{C}$ antibodies were negative. There was no evidence of antiphospholipid syndrome (anti-cardiolipin antibody $\mathrm{IgG}$, $<8 \mathrm{U} / \mathrm{mL}$; anti- $\beta 2$-glycoprotein-I antibody, $<1.2 \mathrm{U} / \mathrm{mL}$; lupus anticoagulant (dilute Russell's viper venom time), 0.98). Echocardiography revealed normal systolic and diastolic function and no signs of pulmonary artery hypertension (the diastolic gradient between right atrium and right ventricle was $17 \mathrm{mmHg}$ ). Abdominal computed tomography (CT) and magnetic resonance imaging (MRI) (Fig. 1) revealed dilatation of the extrahepatic and intrahepatic portal veins and collateral veins, as well as severe splenomegaly, which were compatible with portal hypertension, although no thrombi were found. Endoscopy revealed esophageal varices in the middle to lower esophagus and gastric varices in the cardia

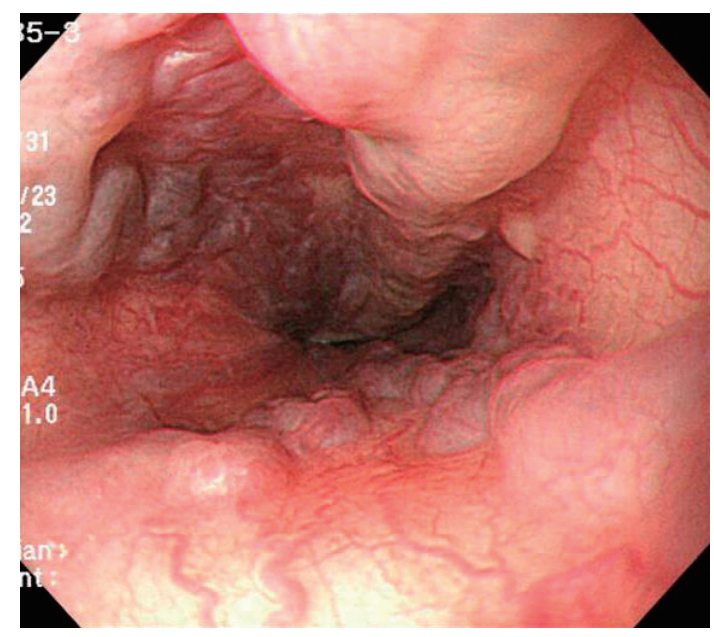

Figure 2. Gastrointestinal endoscopy indicates esophageal varices $(\mathrm{Lm}, \mathrm{F} 2, \mathrm{RC}(+), \mathrm{Cb})$.

(Fig. 2), which were considered to be the cause of the bleeding. She was successfully treated with endoscopic injection sclerotherapy and discharged in late November 2009. During the hospitalization, there were no signs of acute exacerbation of MCTD.

She was admitted to our hospital again because of recurrent severe gastrointestinal tract bleeding in late December 2009. Her laboratory studies on the re-admission showed persistent pancytopenia, mild liver enzyme elevation and low levels and lowered activity of the protein $\mathrm{C}$ antigen: white blood cell count, 2,200/ $\mu \mathrm{L}$ (neutrophils $84.0 \%$, lymphocytes $12.0 \%$, monocytes $2.0 \%$, eosinophils $2.0 \%$ ); red blood cell count, $2.59 \times 10^{6} / \mu \mathrm{L}$; hemoglobin, $7.7 \mathrm{~g} / \mathrm{dL}$; hematocrit, 22.6\%; platelet count, $3.8 \times 10^{4} / \mu \mathrm{L}$; aspartate aminotransferase, $47 \mathrm{IU} / \mathrm{L}$; alanine aminotransferase, $36 \mathrm{IU} /$ L; alkaline phosphatase, 199 IU/L; $\gamma$-glutamyl transpeptidase, $34 \mathrm{IU} / \mathrm{L}$; protein $\mathrm{C}$ antigen level, 54\%; protein $\mathrm{C}$ activity, 58\%; protein $\mathrm{S}$ activity, 78\%; antithrombin III, 93\%; prothrombin time, 16.3 seconds; international normalized ratio, 1.40; activated partial thromboplastin time, 40.4 seconds. Endoscopy revealed recurrence of variceal bleeding in the middle esophagus and cardia, which were treated with endoscopic variceal ligation. The abdominal CT scan confirmed that dilatation of the extrahepatic and the intrahepatic portal vein and collateral veins, and severe splenomegaly remained, although extrahepatic portal vein thrombi were not found. Laparoscopic splenectomy and liver biopsy were performed in late January 2010, and the patient's pancytopenia improved after surgery. Histological examination of the splenic mass showed congestion and hemosiderin-laden macrophages. The liver biopsy specimen showed only nonspecific mild inflammation. Portal vein thrombosis, narrowing of portal veins, nodular regenerative hyperplasia and cirrhosis were not found (Fig. 3). One day after surgery, intrahepatic portal vein thrombosis in the right and left bifurcation to the right anterior superior branch was revealed by abdominal CT. Subsequently the patient was treated with heparin and danaparoid sodium. After confirmation of no re- 
A.

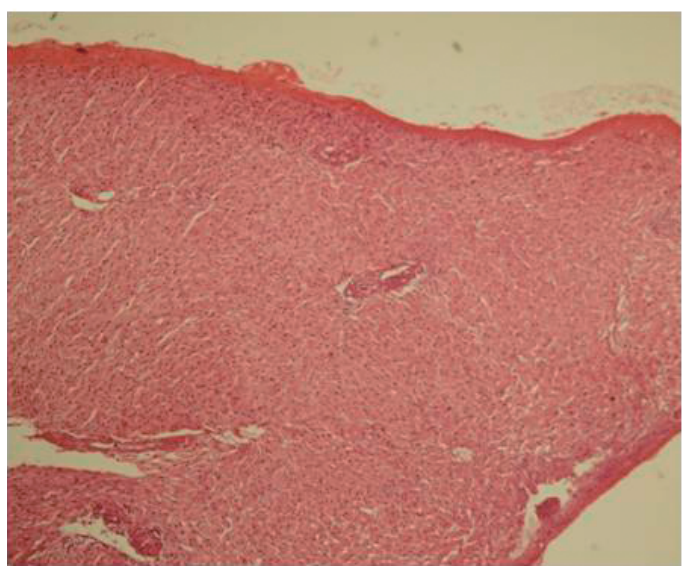

B.

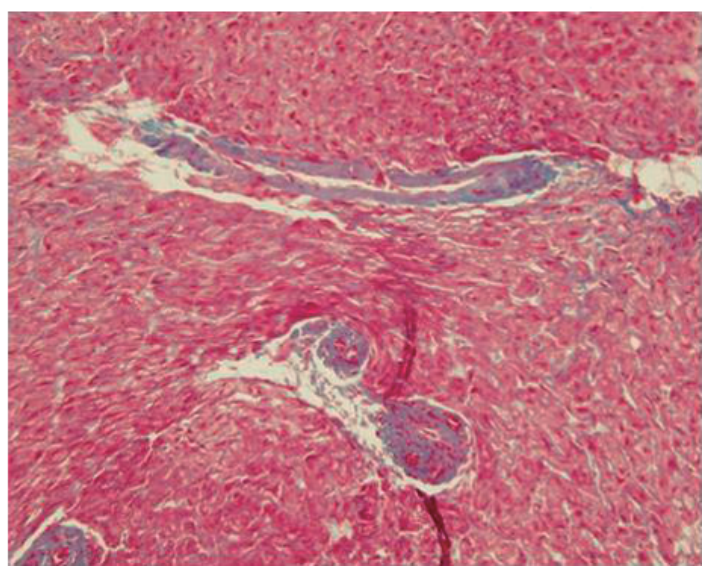

Figure 3. Liver biopsy specimen shows only non-specific mild inflammation, not portal vein obstruction or cirrhosis. A) Hematoxylin and Eosin staining $\times 40$. B) Masson's Trichrome Staining, $\times 100$.

Table 1. Cases of IPH Complicated with MCTD

\begin{tabular}{|c|c|c|c|c|}
\hline Case & Age & Sex & $\begin{array}{l}\text { complicatied diseases } \\
\text { other than IPH or MCTD }\end{array}$ & Refer ence \\
\hline 1 & 64 & $\mathrm{~F}$ & Pulmonary artery hypertension & [3] \\
\hline 2 & 30 & $\mathrm{~F}$ & Pulmonary artery hypertension, SS & {$[4]$} \\
\hline 3 & 49 & $\mathrm{~F}$ & Pulmonary artery hypertension & [5] \\
\hline 4 & 68 & $\mathrm{~F}$ & Chr onic thyroiditis, Pulmonary artery hypertension & [6] \\
\hline 5 & 42 & $\mathrm{~F}$ & Pulmonary artery hypertension & [7] \\
\hline 6 & 41 & $\mathrm{~F}$ & SS & [8] \\
\hline 7 & 29 & $\mathrm{~F}$ & $\mathrm{PCD}$ & presented case \\
\hline
\end{tabular}

Abbreviations: IPH, idiopahic portal hypertension; MCTD, mixed connective tissue disease; $\mathrm{SS}$, Sjögren's syndrome; $\mathrm{PCD}$, protein $\mathrm{C}$ deficiency.

currence of esophagogastric bleeding with a follow-up endoscopy, warfarin therapy was started. After discharge from the hospital, the patient remained in a stable physical condition under treatment with $5.0 \mathrm{mg} /$ day prednisolone and 3.0 mg/day warfarin.

\section{Discussion}

IPH is a clinicopathological condition characterized by splenomegaly, pancytopenia and portal hypertension. Because the etiology of IPH has not been fully determined, the diagnosis of IPH is based on exclusion of other causes, such as extrahepatic portal obstruction or liver cirrhosis. In the present case, PCD was a potential factor in portal vein thrombosis. However, the abdominal CT scan and MRI before splenectomy, and the liver biopsy did not reveal extra- hepatic portal vein thrombosis. In addition, histological examination of the liver did not reveal any apparent causes of portal hypertension, including intrahepatic portal vein thrombosis. Based on the foregoing findings and a clinical course compatible with IPH, we diagnosed the patient with IPH.

Some studies have suggested that IPH is associated with immunological abnormalities. Tokushige et al found that patients with IPH have high serum immunoglobulin levels (1) and activated T cells (2). Several reports have suggested that MCTD plays a role in the pathogenesis of IPH (3-8). It was speculated that persistently elevated inflammatory cytokine levels in sera are correlated with a high risk of endothelial damage in patients with MCTD (9). However, IPH associated with MCTD has rarely been reported; there have only been six reports of IPH in MCTD patients (3-8) (Table 1), and five of the six cases were complicated by esophageal 
varices. The liver biopsy findings of these six cases revealed inflammatory cell infiltration and mild or severe fibrosis in the portal areas, combined with narrowing or obstruction of the portal vein. In the present case, increased activity of MCTD was not observed when esophageal varices rupture developed. However, highly elevated ESR levels persisted under steroid and infliximab therapy whereas joint symptoms had improved. It is speculated that chronic inflammation induced by MCTD affects the portal vein, resulting in microvascular endothelial damage.

An interesting feature of this case is that the patient's clinical condition was complicated by PCD, in addition to MCTD. Protein $\mathrm{C}$ is a vitamin K-dependent coagulation factor, which is activated by thrombin bound to thrombomodulin. Activated protein $\mathrm{C}$ serves as an anticoagulant by regulating the activity of coagulation factors Va and VIIIa in the presence of its cofactor, protein S. Inherited coagulation disorders, including PCD and protein S deficiency, are well known causes of venous thrombosis. We could not know whether PCD in this patient existed before the referral to our hospital. However, most congenital PCD patients develop embolic symptoms after reaching adulthood. In this case, intrahepatic portal vein thrombi were generated after surgery. It is speculated that she had congenital PCD and surgical invasion induced activation of the clotting system, and portal vein thrombogenesis was not sufficiently prevented because of the decrease in the anticoagulation effect of protein C. Harmanci and Bayraktar reported that protein $\mathrm{C}$ and $\mathrm{S}$ deficiencies were found in $13 \%$ of their population of patients with IPH, and suggested the "repetitive microthrombosis theory" in IPH complicated by protein C and S deficiencies; microthrombi in the small intrahepatic branches of the portal vein caused periportal fibrosis-like reconstruction, and gradually resulted in portal vein hypertension, splenomegaly and esophagogastric varices (10). In addition, recent studies suggested that activated protein $\mathrm{C}$ had not only an anticoagulatory function, but also anti-inflammatory and antiapoptotic effects. Activated protein $\mathrm{C}$ binding to the endothelial protein $\mathrm{C}$ receptor activates protease activated receptor-1, which activates intracellular $G$ proteins, and these proteins induce anti-inflammatory and antiapoptotic effects (11). Conversely, PCD can become a risk factor for endothelial cell damage and apoptosis. It is speculated that the decrease in the anti-inflammatory and antiapoptotic effects of activated protein $\mathrm{C}$ aggravates portal endothelium damage, although histological findings did not show evidence to prove this theory. We postulate that synergistic effects of MCTD and PCD induced chronic endothelial dysfunction in this patient, resulting in IPH and esophageal varices.

This is the first report of IPH linked to PCD in a MCTD patient. Both MCTD and PCD are causes of systemic endothelial damage. It is therefore necessary to recognize that synergy of PCD and MCTD may trigger severe vessel disorders, such as IPH and esophagogastric variceal rupture. This case is an interesting case for consideration of the role of autoimmune diseases and coagulation abnormalities in the pathogenesis of IPH.

Conflict of interests: The authors declare that they have no conflict of interests.

\section{References}

1. Tokushige K, Komatsu T, Ohzu K, Yamauchi K, Obata H. A defective autologous mixed lymphocyte reaction in patients with idiopathic portal hypertension. J Gastroenterol Hepatol 7: 270-273, 1992.

2. Tokushige $\mathrm{K}$, Hirose $\mathrm{S}$, Nishimura $\mathrm{H}$, et al. Abnormal $\mathrm{T}$ cell activation and skewed $\mathrm{T}$ cell receptor $\mathrm{V}$ beta repertoire usage in Japanese patients with idiopathic portal hypertension. Clin Immunol Immunopathol 75: 206-213, 1995.

3. Sawai T, Tutikawa K, Watanabe T, Kyogoku M. A case of mixed connective tissue disease (MCTD) complicating pulmonary hypertension and portal hypertension (in Japanese). Ryumachi 28: 164169, 1988 (in Japanese, Abstract in English).

4. Muryoi T, Harata N, Tamata E, Sasaki T, Yoshinaga K. A case of mixed connective tissue disease with idiopathic portal hypertension. Nippon Naika Gakkai Zasshi 78: 984-985, 1989 (in Japanese, Abstract in English).

5. Ozawa T, Nakagawa O, Hasegawa $S$, et al. A case of mixed connective tissue disease accompanied with idiopathic portal hypertension and pulmonary hypertension. Nippon Naika Gakkai Zasshi 78: 1799-1800, 1989 (in Japanese, Abstract in English).

6. Hirasaki S, Koide N, Ogawa H, et al. Mixed connective tissue disease associated with idiopathic portal hypertension and chronic thyroiditis. J Gastroenterol 32: 808-811, 1997.

7. Sekiguchi Y, Amano K, Takano Y, et al. Portal and pulmonary hypertension in a patient with MCTD. Ryumachi 39: 657-663, 1999 (in Japanese, Abstract in English).

8. Rai T, Ohira H, Fukaya E, et al. A case of merged idiopathic portal hypertension in course of mixed connective tissue disease. Hepatol Res 30: 51-55, 2004.

9. Bodolay E, Aleksza M, Antal-Szalmás P, et al. Serum cytokine levels and type 1 and type 2 intracellular $\mathrm{T}$ cell cytokine profiles in mixed connective tissue disease. J Rheumatol 29: 2136-2142, 2002.

10. Harmanci O, Bayraktar Y. Clinical characteristics of idiopathic portal hypertension. World J Gastroenterol 13: 1906-1911, 2007.

11. Dahlbäck B, Villoutreix BO. Regulation of blood coagulation by the protein $\mathrm{C}$ anticoagulant pathway: novel insights into structurefunction relationships and molecular recognition. Arterioscler Thromb Vasc Biol 25: 1311-1320, 2005.

(C) 2010 The Japanese Society of Internal Medicine

http://www.naika.or.jp/imindex.html 